Wachsendes Umweltbewusstsein und steigende Energiepreise lassen Eigenheimbesitzer verstärkt über Energieeinsparmaßnahmen nachdenken. Im norddeutschen Raum ist das zweischalige Außenmauerwerk mit dazwischen liegender Luftschicht die übliche Bauweise. Eine schnelle und kostengünstige Möglichkeit der energetischen Sanierung bietet die nachträgliche Hohlraumdämmung des zweischaligen Außenmauerwerks, indem die Luftschicht vollständig mit Dämmstoff verfüllt wird.

Die Funktion der Luftschicht - das Belüften und Entfeuchten der Mauerwerkskonstruktion - wird durch diese Maßnahme aufgehoben. Darin könnte ein Schadenspotenzial (Feuchte) des Verfahrens gesehen werden.

Um die Auswirkungen und die möglichen Risiken der nachträglichen Hohlraumdämmung zu untersuchen, wurde an der Jade Hochschule in Oldenburg im Fachgebiet Baustofftechnologie und Bauwerkserhaltung über einen Zeitraum von zwei Jahren ein Forschungsvorhaben mit dem Thema "Nachträgliche Hohlraumdämmung - Anwendung und Dauerhaftigkeit" durchgeführt. Die genaue Festlegung bzw. die Überprüfung der Randbedingungen, bei denen eine nachträgliche Hohlraumdämmung unter bauphysikalischen Gesichtspunkten möglich ist, stellte den Schwerpunkt dieses Vorhabens dar. In diesem Zusammenhang wurden Materialdaten der Dämm- und Baustoffe im Labor ermittelt und anhand dieser Parameter numerische Simulationen zum Feuchte- und Wärmeverhalten des gedämmten Mauerwerks durchgeführt. Außerdem wurde in einem Feldver- such die Wasseraufnahme und das Setzungsverhalten der Dämmstoffe überprüft. Zudem erfolgten Untersuchungen an Gebäuden vor und nach dem Einbringen einer Hohlraumdämmung. Die Ergebnisse dieses Forschungsvorhabens werden im vorliegenden Leitfaden zusammengefasst.

Ziel dieses Leitfadens ist es, eine Übersicht über die Möglichkeiten und Grenzen einer nachträglichen Hohlraumdämmung des Außenmauerwerks zu geben. Dies beinhaltet Anweisungen und Empfehlungen zu Voruntersuchungen am Gebäude sowie vorbereitende Maßnahmen bei der Durchführung und der anschlieBenden Qualitätskontrolle der Dämmmaßnahme.

Aufgrund der starken Nachfrage der Erstauflage (2010) liegt nun die zweite, überarbeitete Auflage vor. Neben einigen kleineren Überarbeitungen wurde diese Auflage um eine Auflistung derzeit zugelassener Dämmstoffe erweitert (Stand Juli 2012). Der Leitfaden soll Arbeitsgrundlage für Energieberater, Architekten, Ingenieure, ausführende Firmen sowie Eigenheimbesitzer sein.

\section{Das Projektteam}




$\begin{array}{ll}\text { Vorteile } & \\ \text { Hohlraumdämmung spart Energie ... } & 2 \\ \text { _.. und steigert den Wohnkomfort } & 3 \\ \text { Dämmstoffe } & \\ \text { Dämmstoffgruppen } & 5 \\ \text { Anforderungen an die Dämmstoffe } & 7 \\ \text { Bestandsuntersuchungen } & \\ \text { Warum Bestandsuntersuchungen } & 10 \\ \text { Zustand der Vormauerschale } & 11 \\ \text { Zustand der Luftschicht und der Mauerwerksanker } & 12 \\ \text { Undichtigkeiten an den Mauerschalen } & 12 \\ \text { Weitere Besonderheiten am Gebäude } & 12 \\ \text { Ergänzende Untersuchungen } & \\ \text { Wärmebrücken } & 13 \\ \text { Feuchteverhalten der Wand } & 14 \\ \text { Ausführung } & \\ \text { Abdichten der Hohlschicht } & 15 \\ \text { Dämmen über das Dach } & 15 \\ \text { Anordnung der Einblasöffnungen } & 16 \\ \text { Dämmen über das Kleinlochverfahren } & 16 \\ \text { Dämmen über das Großlochverfahren } & 16 \\ \text { Qualitätskontrolle } & 17 \\ \text { Dämmstoffe und ihre Eigenschaften } & 18 \\ \text { Impressum } & 19 \\ \text { Checkliste } & 20\end{array}$

